\title{
Hot Topics in Functional Neuroradiology
}

\author{
S.H. Faro, F.B. Mohamed, J.A. Helpern, J.H. Jensen, K.R. Thulborn, I.C. Atkinson, H.I. Sair, and D.J. Mikulis
}

\begin{abstract}
SUMMARY: Functional neuroradiology represents a relatively new and ever-growing subspecialty in the field of neuroradiology. Neuroradiology has evolved beyond anatomy and basic tissue signal characteristics and strives to understand the underlying physiologic processes of central nervous system disease. The American Society of Functional Neuroradiology sponsors a yearly educational and scientific meeting, and the educational committee was asked to suggest a few cutting-edge functional neuroradiology techniques (hot topics). The following is a review of several of these topics and includes "Diffusion Tensor Imaging of the Pediatric Spinal Cord"; "Diffusional Kurtosis Imaging”; "From Standardization to Quantification: Beyond Biomarkers toward Bioscales as Neuro MR Imaging Surrogates of Clinical End Points"; Resting-State Functional MR Imaging”; and "Current Use of Cerebrovascular Reserve Imaging."
\end{abstract}

ABBREVIATIONS: CVR = cerebrovascular reserve; DKI = diffusional kurtosis imaging; FA = fractional anisotropy; ISNSCI = Standards for Neurologic Classification of Spinal Cord Injury; MD = mean diffusivity; rs-fMRI = resting-state $\mathrm{fMRI}$; $\mathrm{CCI}=$ spinal cord injury

\section{DIFFUSION TENSOR IMAGING OF THE PEDIATRIC SPINAL CORD \\ Scott Faro, MD, and Feroze Mohamed, PhD; Department of Radiology, Temple University School of Medicine, Philadelphia, Pennsylvania}

Diffusion tensor imaging is a relatively new noninvasive MR imaging technique that quantifies the multidirectional diffusion of water molecules. There are many applications of DTI in the brain, and there is rising interest in exploring DTI applications in the spine. The unique characteristic architecture of the spinal cord is that its highly anisotropic nature may allow DTI to localize white matter, separate white from gray matter, and assess structural pathologic changes of the cord. Recent work has shown the clinical utility of DTI in spinal cord compression and cervical myelopathy in adults. ${ }^{1,2}$ Pediatric DTI has also been explored recently, and several advances have been made in the field of DTI of pediatric spinal cord injury (SCI). ${ }^{3,4}$ A variety of DTI indices has been explored, including fractional anisotropy (FA), mean diffusivity (MD), radial diffusivity, and axial diffusivity. Spinal cord injury occurs in both adults and children, with an estimated 11,000 cases occurring yearly in the United States. In this review, we will specifically look at the importance of DTI in pediatric SCI.

Currently, the evaluation and classification of neurologic im-

Please address correspondence to Scott H. Faro, MD, Department of Radiology, Temple University School of Medicine, 3401 N. Broad St, Philadelphia, PA 19140; e-mail: faros@tuhs.temple.edu

http://dx.doi.org/10.3174/ajnr.A3721 pairment in adults and children with SCI are assessed by using the International Standards for Neurologic Classification of Spinal Cord Injury (ISNCSI). These standards involve testing of motor and sensory functions of the limbs, trunk, rectum, and anorectal area and are used to predict recovery of neurologic function, plan treatment, and determine treatment effectiveness. The ISNSCI have relatively low utility in the pediatric population and can lead to unreliable assessment of neurologic abnormalities. An objective assessment of SCI may provide important information complementary to conventional clinical and imaging evaluation and may lead to a more accurate prognosis of recovery from SCI.

DTI of the spinal cord is technically limited by various factors. Specifically in pediatric populations, the small cord volume (approximately $1 \mathrm{~cm}$ in diameter) leads to a low SNR. Cerebral spinal fluid pulsation and blood flow can produce prominent ghosting artifacts and degrade image quality. The spinal cord is also subject to respiratory and cardiac movements, which cause image blurring and increased or decreased signal intensity. Different tissue interfaces (bone, soft tissue, or fluid) can create susceptibility artifacts. Swallowing or related motion artifacts are mostly seen when imaging the cervical spinal cord. Finally, in pediatric imaging especially, the possibility of increased subject motion makes obtaining accurate and reproducible DTI parameter values difficult. Artifact-reducing techniques can be used to overcome some of these issues. However, particularly in pediatric imaging, these techniques are not without challenges. Cardiac gating and respiratory compensation may increase acquisition time, and sedation 


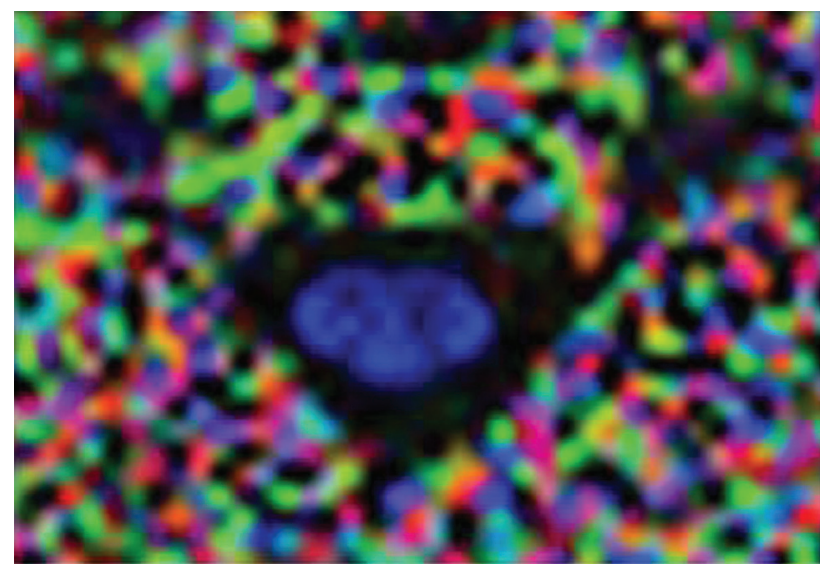

FIG 1. Axial color FA map of a normal midcervical spine in a healthy pediatric subject.

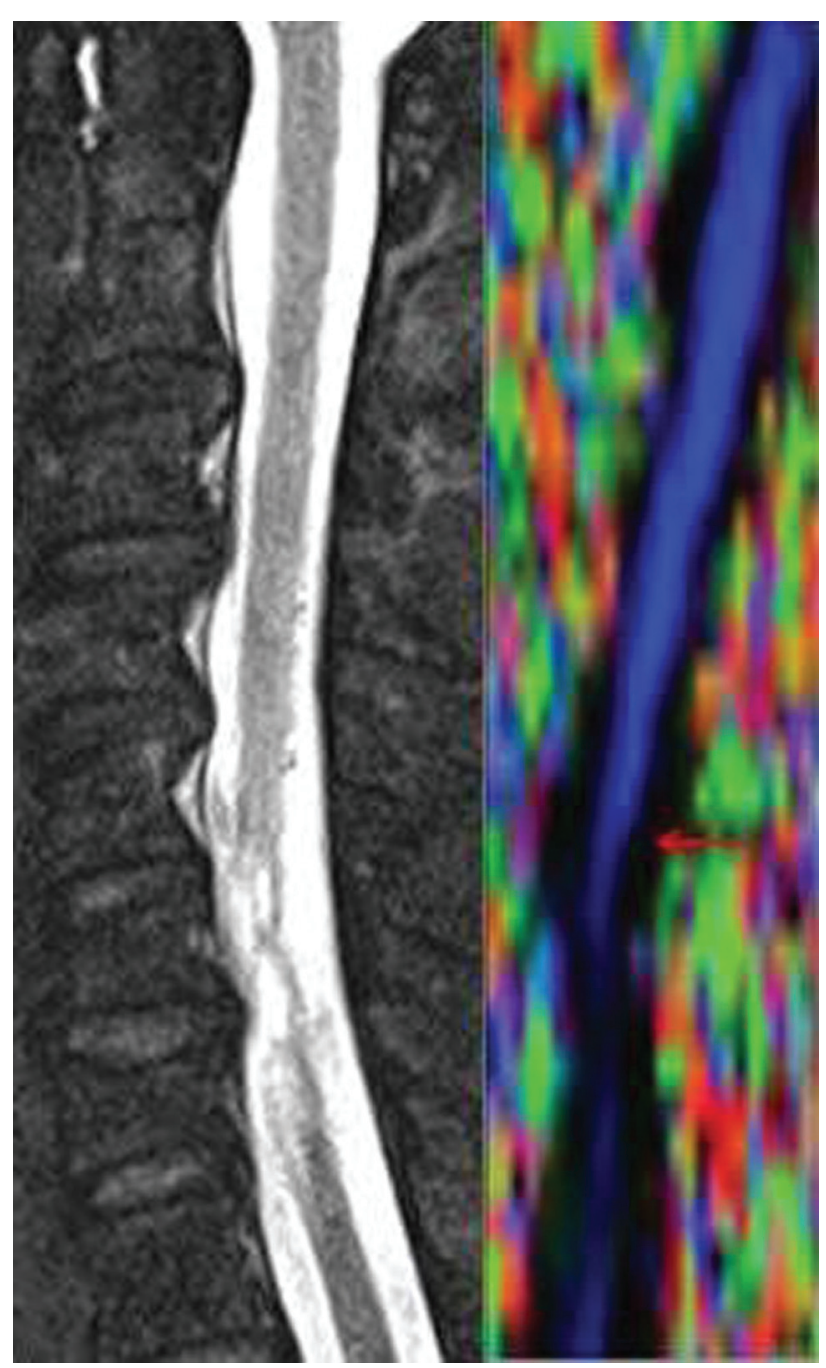

FIG 2. Color FA map of a patient with $\mathrm{SCl}$ (right) and a corresponding T2-weighed sagittal image (left) demonstrating $\mathrm{SCl}$ at the $\mathrm{C5}$ level (arrow).

is typically not desirable in children. Thus, fast, reliable, and highresolution imaging is needed to image pediatric subjects.

In Figs 1 and 2, we show the use of an inner FOV DTI imaging based on 2D radiofrequency excitations of the pediatric cervical spinal cord. Figure 1 shows an axial color FA map of a healthy pediatric patient, which demonstrates very good gray/white matter differentiation and cord/CSF conspicuity. Figure 2 shows a sagittal color FA map and a TSE T2 image of a pediatric patient with a spinal cord injury, demonstrating a change in FA signal that corresponds to a lower cervical injury. Figure 3 shows a graphic representation of the DTI indices (FA, MD, radial diffusivity, and axial diffusivity) through the cervical spine in a pediatric patient with a focal C6 SCI. This graph shows, in addition to focal changes in DTI indices in the lower cervical spine (decreased FA, increased $\mathrm{MD}$, radial diffusivity, and axial diffusivity), diffusion changes throughout the cord above the level of injury compared with a healthy control. The cervical cord above the injury did not demonstrate any abnormal signal on the T2-weighted images. This technique has been shown to be very useful in generating reliable and repeatable DTI data from the pediatric spinal cord and is a promising technique for quantification of SCI.

DTI will help our understanding of the pathophysiology of SCI and may give added information related to the clinical care of patients with SCI. Preliminary work has shown good correlation of ISNSCI scoring with DTI indices in these patients. ${ }^{5}$ Research related to pediatric patients can be translatable to adult patients with SCI. Continued research by using DTI is needed to assess neurologic damage after traumatic SCI and to improve care, treatment, and rehabilitation methods.

\section{DIFFUSIONAL KURTOSIS IMAGING \\ Joseph A. Helpern, PhD, and Jens H. Jensen, PhD; Department of Radiology and Radiological Science, Medical University of South Carolina, Charleston, South Carolina}

The nominal resolution of human brain MR imaging is typically no more than a millimeter at best. This level of resolution provides excellent anatomic images, but the cellular microstructure, with characteristic lengths of tens of microns, is essentially invisible. This is a substantial limitation because disease processes often manifest themselves most clearly in terms of microstructural changes; this shortcoming is precisely the reason histopathology plays such a prominent role in the diagnosis of numerous maladies.

A powerful and elegant MR imaging method for investigating cellular microstructure is diffusion MR imaging. Diffusion MR imaging yields information about cellular microstructure due, in part, to a fortuitous matching of typical cellular sizes with the water diffusion lengths in biologic tissues during the time when the diffusion gradients are applied. Thus diffusing water is a natural reporter molecule for probing cellular microstructure.

To date, the leading MR imaging technique used to assess brain tissue microarchitecture is diffusion tensor imaging. Although DTI is powerful, it yields only a fraction of the potential information possible because it assumes that all water diffusion is Gaussian, even though we know it is not. In fact, by neglecting diffusional nonGaussianity, DTI discards much of the microstructural information that diffusion MR imaging is, in principle, capable of detecting.

To have a diffusion MR imaging method that maintains the practical advantages of DTI (ease of implementation, time efficient) but also allows diffusional non-Gaussianity to be quantified, our group has developed an extension of DTI called diffu- 

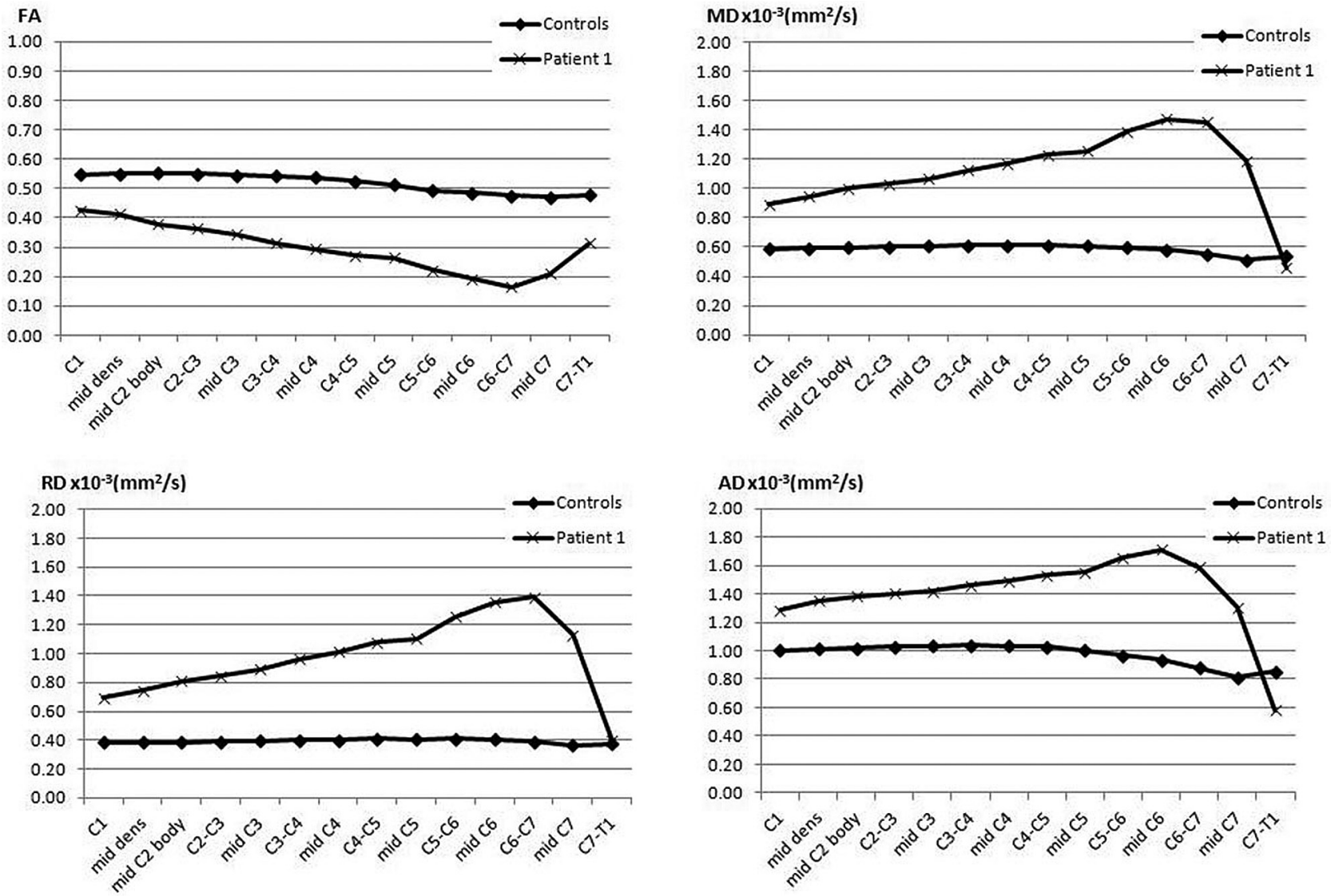

FIG 3. Healthy control and a patient with $\mathrm{SCl}$ at the mid-C6 level displaying changes in various DTI indices (FA, MD, radial diffusivity, and axial diffusivity) from $\mathrm{Cl}$ to $\mathrm{C} 7$.

sional kurtosis imaging (DKI). ${ }^{6-8}$ Kurtosis is a well-established and widely used dimensionless statistical measure for quantifying the non-Gaussianity of arbitrary probability distributions, like that of diffusion. The significance of this approach is that diffusional non-Gaussianity effects are natural indicators of tissue microarchitectural complexity (ie, integrity) and may be used to improve tissue characterization, including changes associated with disease processes.

Since its introduction in 2005, DKI has been used to investigate several neuropathologies including brain cancer, ${ }^{9,10}$ prostate cancer,${ }^{11}$ ischemic stroke, ${ }^{12,13}$ attention deficit/hyperactivity disorder, ${ }^{14}$ epilepsy, ${ }^{15}$ Huntington disease, ${ }^{16}$ brain trauma, ${ }^{17}$ and spondylotic myelopathy. ${ }^{18}$ For brain cancer, 2 prior studies ${ }^{9,10}$ indicate that the additional information provided by DKI may improve the sensitivity and specificity for grading gliomas. For ischemic stroke, ${ }^{12,13}$ large changes of $50 \%-100 \%$ in diffusional kurtosis may occur within ischemic lesions, suggesting profound changes in microstructural complexity. In addition, mismatches in the regions with decreased diffusivity and those with increased kurtosis are sometimes observed, suggesting that the 2 metrics are quantifying distinct microstructural changes. For brain trauma, ${ }^{17}$ diffusional kurtosis has demonstrated sensitivity to microstructural damage exceeding that of conventional diffusion metrics obtainable with DTI. Figure 4 shows the mean diffusivity and mean kurtosis maps of a patient with subacute ischemic stroke. Notice the distinct ischemic lesion signal heterogeneity on mean kurtosis, which is not apparent on MD maps (red arrows).

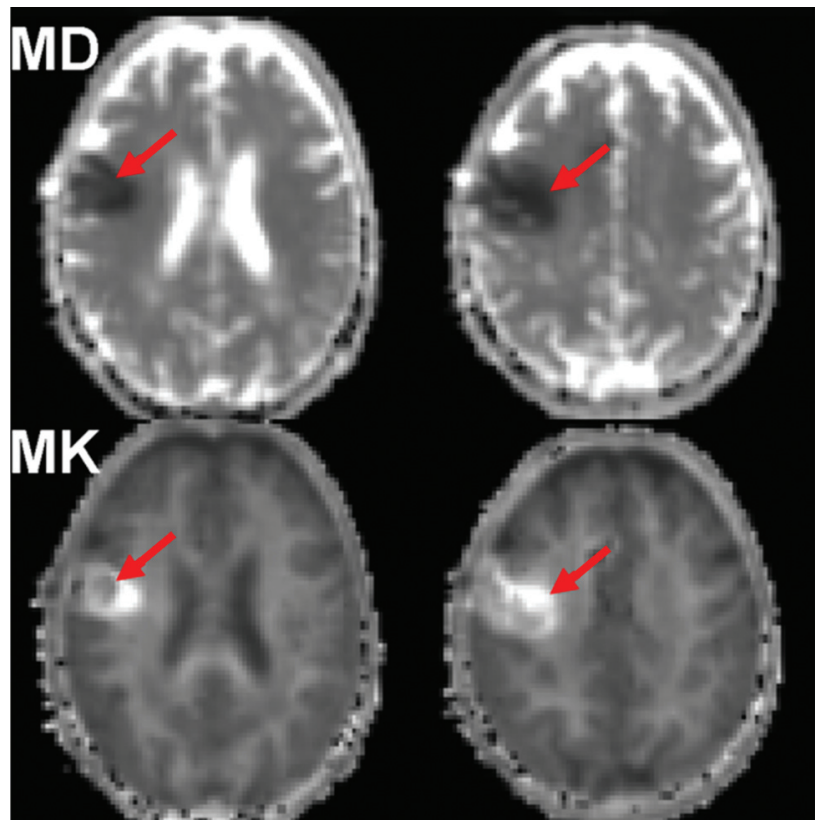

FIG 4. Mean diffusivity and mean kurtosis maps of a patient with subacute ischemic stroke. Notice the distinct ischemic lesion signal heterogeneity on mean kurtosis that is not apparent on MD maps (red arrows).

With standard clinical scanners and pulse sequences, highquality, whole-brain DKI datasets may now be acquired within 7 minutes, making feasible its incorporation into many clinical and 
Features for a bioscale that enable its role as a surrogate for a clinical end point beyond the NIH definition of a biomarker

\begin{tabular}{|c|c|c|}
\hline No. & Properties of a Bioscale & Implications \\
\hline 1 & Image-derived map of the disease site & More sensitivity to earlier disease than diluted remote biomarkers \\
\hline 2 & Spatially resolved quantitative parameter & Precise and accurate measurement \\
\hline 3 & Small biologic variance in the healthy population & Sensitivity to early disease \\
\hline 4 & $\begin{array}{l}\text { Continuously and monotonically varying with } \\
\text { disease progression }\end{array}$ & Sensitivity to disease progression or response to treatment \\
\hline 5 & Intrinsically related to the disease mechanism & $\begin{array}{l}\text { Highly conserved metabolic parameter, essential for use as a } \\
\text { surrogate of a clinical end point }\end{array}$ \\
\hline
\end{tabular}

Note:-NIH indicates National Institutes of Health.

research protocols. Moreover, the application of DKI obviates DTI, because DKI provides all the metrics obtainable from DTI and with improved accuracy. Thus, upgrading from DTI to DKI only adds a few minutes of additional scanning time. In the near future, the imaging times for diffusion MR imaging in general, and DKI in particular, are likely to be substantially improved with the advent of multiband echo-planar sequences. Thus, the acquisition time will not be a significant obstacle for the widespread adoption of DKI. The DKI sequence is now a work in progress of Siemens Health Care (Erlangen, Germany) and is currently being tested at several medical centers worldwide. To learn more about DKI and to download free software for analysis, please go to http://academicdepartments.musc.edu/cbi/dki/.

\section{FROM STANDARDIZATION TO QUANTIFICATION: BEYOND BIOMARKERS TOWARD BIOSCALES AS NEURO MR IMAGING SURROGATES OF CLINICAL END POINTS \\ Keith R. Thulborn and Ian C. Atkinson; Center for Magnetic Resonance Research, University of Illinois at Chicago, Chicago, Illinois}

The term "biomarker" has been defined by the National Institutes of Health as "a characteristic that is objectively measured and evaluated as an indicator of normal biologic processes, pathogenic processes, or pharmacologic responses to a therapeutic intervention." Biomarkers have conventionally been genes, gene products, or metabolites that are obtained from bodily fluids, often remote from the site of disease and measured as concentrations by using standard analytic techniques that have established accuracies. The use of such nonimaging biomarkers as surrogates for clinical end points (eg, high blood pressure is an early indicator for cardiac disease) has a long history that is encapsulated statistically in the controversies of the Prentice criterion. ${ }^{19}$ The validation of a biomarker as equivalent to a true outcome is nontrivial because that validity requires a full trial that makes the need for the subsequent use of a biomarker questionable in some circumstances. ${ }^{20}$ In fact, the reality is that even the most highly cited biomarkers introduced into medicine from single studies fail to prove useful in subsequent meta-analyses. ${ }^{21}$ Despite these concerns, the incentive for pursuing surrogate measures of a true clinical end point is the possibility of shortening durations and decreasing population sizes required for clinical trials.

Despite these controversial issues of biomarker validity, imaging characteristics have been proposed as potential biomarkers. Imaging parameters are typically arbitrary signal-intensity scales that require the acquisition procedures to be quantified if the scale is to be compared during a certain time or between patients.
"Quantification" is a term that contains the concept of accuracy relative to a known standard and has dimensional units.

For imaging to provide a useful surrogate end point for clinical trials, the imaging characteristic must be calibrated to establish its distribution (mean, variance) in the normal population. Ideally, the biologic variance should be small. Because the progression from healthy to illness is usually continuous, any parameter measuring this progression should also be continuous. A parameter that continuously reflects normal to progressively worsening disease is unlikely to discriminate early disease unless it undergoes changes that are large compared with the normal variance. This possible outcome is an expression of the Prentice criterion, ${ }^{19}$ in that the surrogate parameter must be related mechanistically to the progression of the disease. This simple rationale suggests some features that are needed for an imaging parameter to be useful as a surrogate of a clinical end point (Table). Imaging potentially offers sensitivity to early disease by having direct access to the site of disease rather than being dependent on the release of biomarker molecules into bodily fluids that are only sampled remotely after dilution.

The signal intensity of clinical MR imaging is not only a reflection of the concentration and nuclear properties of the hydrogen nuclei in the patient but is highly dependent on acquisition parameters and equipment performance. The nuclear properties of protons, expressing the nuclear and electronic dipolar interactions of the hydrogen atoms in water and fat, as summarized by the longitudinal and transverse relaxation parameters, only distantly reflect the biology of the tissue. The MR signal from water reflects the tissue milieu rather than metabolic reactions. However, high concentrations are required to generate sufficient MR signal for spatial encoding into an image at a relevant resolution in an acceptable acquisition time for patients. This requirement limits metabolic observations. Although MR imaging is remarkably good at displaying anatomy, especially of the brain, anatomy is not a useful surrogate of early disease because of its wide biologic variance. For example, brain volume decreases with age in cognitively healthy subjects and in patients with dementia. Although, on average, patients with dementia have smaller brain volumes than healthy subjects, brain volumes vary greatly and have considerable overlap between these groups so that brain volume does not allow an individual patient to be classified confidently into either category. This example illustrates that useful surrogates must not only be measured accurately but also have small biologic variance to provide sensitivity for the detection of early disease and its progression. In contrast to the desirable diversity (ie, variance) of anatomy that drives evolution, a small biologic variance suggests the need for a parameter that is highly conserved and vital for biologic health. 


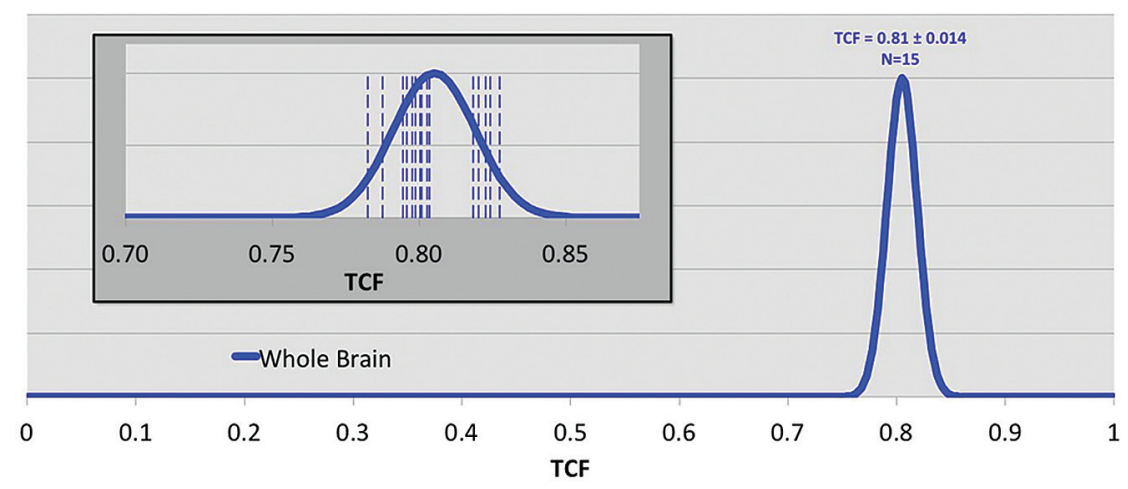

FIG 5. Distribution of tissue cell fraction measured in brain parenchyma for the whole brain, including gray and white matter (blue, $0.81 \pm 0.014$ ) for cognitively healthy individuals $(n=15)$. The inset shows the individual measurements (vertical dashed lines) of each individual. The SD of the distribution of cell attenuation in healthy subjects is $<2 \%$ of the total range of possible cell densities.

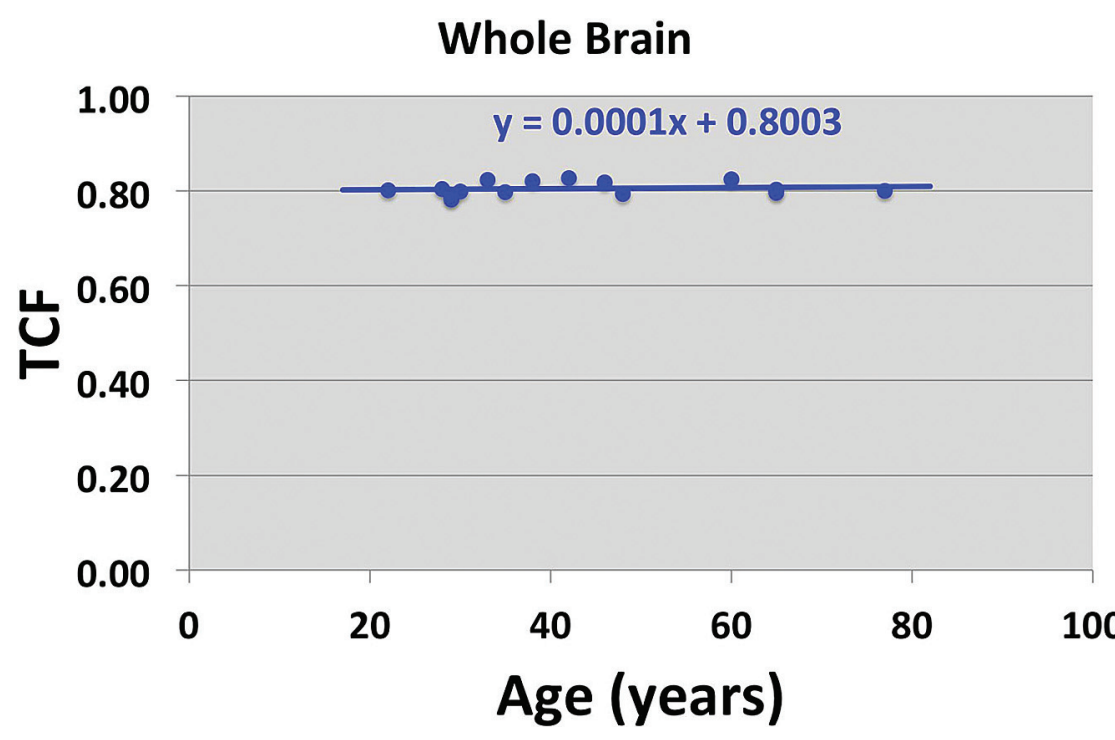

FIG 6. Tissue cell fraction as a function of age (years) in the cognitively healthy subjects in Fig 1. The fitted line has a near-zero gradient, indicating that there is no age dependence for mean kurtosis in the whole brain of cognitively healthy individuals.

A pertinent example of a highly conserved metabolic process is sodium ion homeostasis in the brain. ${ }^{22}$ This tightly regulated process is controlled by systemic, tissue, and cellular events to ensure a stable resting membrane potential across the neuron cell membrane that fuels the action potentials of brain function. Sodium MR imaging can quantify this homeostatic process by measuring the tissue sodium concentration, TSC, and its derived equivalent of tissue cell fraction, $\mathrm{TCF}^{23}$ These parameters can be measured precisely with an experimental error of approximately 5\% after B0 and B1 corrections and calibration in $<30$ minutes of patient time at $3 \mathrm{~T}$ at a nominal isotropic spatial resolution of $5 \mathrm{~mm}$. The mean kurtosis parameter has a narrow variance, as shown in Fig 5, and may be very sensitive to hippocampal cell loss in mild cognitive impairment and probably Alzheimer disease. Its independence of subject age in cognitively healthy individuals, as shown in Fig 6, indicates the importance of cell density for normal cognitive function. An operational definition of cell viability is its ability to maintain the integrity of its cell membrane. Examples of the sensitivity of tissue sodium concentration and mean kurtosis measurements in the settings of acute stroke and treatment response of brain tumors have been published. ${ }^{24,25}$

Rather than use the term "imaging biomarker," which refers to a descriptive risk factor for predicting clinical outcomes, the term "bioscale" has been coined to reflect the features of an imaging-based parameter needed to be a useful surrogate marker in clinical states. A bioscale is a continuous variable that is quantified against a standard with a defined accuracy. The bioscale has a small range (small variance) of normal values indicating its fundamental importance for health. A bioscale should directly reflect the cellular processes that are disrupted by the disease, as required by the validity criteria. ${ }^{20}$ Bioscale encompasses the concept of noninvasive spatial quantification that is available in an image of the site of the disease. As the imaging parameter is mechanistically linked to the disease process, the validity criteria that underlie the concept of surrogate markers are addressed. The statistical requirements of surrogate markers of clinical outcomes suggest that metabolic imaging by using nuclei (sodium 23) other than protons may have added value, especially now that there are solutions to the lower intrinsic sensitivity of these nuclei at higher field strengths. ${ }^{26}$

\section{RESTING-STATE FUNCTIONAL MR IMAGING \\ Haris Sair, MD; Department of Radiology, Johns Hopkins School of Medicine, Baltimore, Maryland}

Resting-state functional MR imaging (rsfMRI) has emerged as a novel tool to supplement task-based fMRI in the evaluation of brain function. Spontaneous low-frequency fluctuations in regional cerebral blood flow have been demonstrated with blood oxygen level-dependent imaging, occuring at a frequency of approximately $0.01-0.1 \mathrm{~Hz}^{27}$; these spatially distinct, however temporally synchronous, fluctuations constitute "functionally related" brain areas, whose regions form intrinsic brain networks. ${ }^{28,29}$ A relatively consistent set of reproducible brain networks has been demonstrated by various groups. ${ }^{30,31}$ Although a universal set of intrinsic networks has not been agreed on, several major networks have been investigated in detail, including the motor network, language network (Fig 7), and the default mode network, the most studied of the these networks.

There are several advantages of using rs-fMRI in contrast to traditional task-based fMRI. First, these intrinsic low-frequency fluctuations account for a large percentage of blood oxygen leveldependent modulation, even in task-based fMRI experiments, thereby allowing higher sensitivity in signal detection. ${ }^{32}$ Second, a limitation of task-based fMRI is the necessity of designing a spe- 


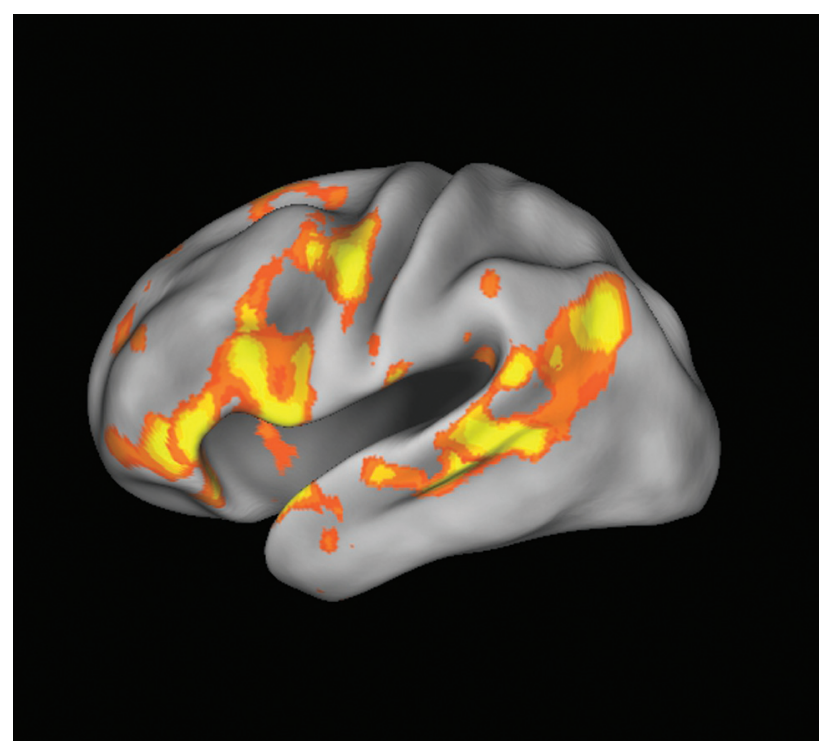

FIG 7. Language network extracted from resting-state fMRI by using independent component analysis in a single healthy control demonstrates Broca and Wernicke areas.

cific task paradigm to interrogate changes associated with a specific neurobehavioral response. No specific task is designated in rs-fMRI (other than instructions on keeping the eyes either closed or open, the latter either fixated on a cross-hair or allowing random eye motion). Third, related to the second advantage is the fact that in many situations, a subject may not be able to perform the task adequately to elicit a reliable blood oxygen level-dependent modulation response. This scenario can occur in various circumstances, for example, in imaging children, debilitated patients, and those whose native language is different from the that in the paradigm used. Last, rs-fMRI has the potential benefit of allowing shorter imaging time to generate more information compared with task fMRI. For example, in clinical presurgical mapping in which motor and language tasks are used, there may be a variety of paradigms used in task fMRI, with several of them repeated to ensure reliability. Although the optimum scanning time for reliable network mapping in individual subjects using rs-fMRI is still being investigated, at the group level, approximately 6-7 minutes of scanning time has been shown to be adequate for reliable network detection. ${ }^{31}$ Furthermore, instead of being able to generate only one specific domain such as languagerelated brain areas, the benefit of rs-fMRI is that multiple brain networks may be generated using a single run.

In analyzing functional connectivity at rest, several approaches are used, the most common being region of interestbased correlations, as well as model-free analysis using independent component analysis or clustering. ${ }^{33}$ In addition to connectivity within the respective intrinsic brain networks, information regarding between-network correlations also yields important clues in normal brain function and disruption of those relationships in disease. ${ }^{34}$

Disruptions of brain networks have been demonstrated in various diseases, including Alzheimer disease, major depression, and epilepsy, to name a few. Although the list of findings in rs-fMRI pertaining to disease is extensive and beyond the scope of this article, several categories of clinical rs-fMRI applications can be demonstrated. First, rs-fMRI may serve as an adjunct tool for clinical or preclinical diagnosis. As an example of patients with cognitive disorders, a difference in default mode network connectivity was demonstrated in patients with mild cognitive impairment who eventually converted to Alzheimer disease compared with mild cognitive impairment nonconverters. ${ }^{35}$ Rs-fMRI may also allow better subsegmentation of heterogeneous diseases such as schizophrenia or the various dementias ${ }^{36}$ or identification of patients in whom pharmacologic intervention may or may not be successful on the basis of intrinsic connectivity before treatment. ${ }^{37}$ Furthermore, rs-fMRI may be used as a potential biomarker for outcome, for example, in the setting of traumatic brain injury. An especially important use of rsfMRI, presurgical mapping, is emerging, in particular in epileptogenic localization $^{38}$ or in brain tumors. ${ }^{39}$

Although the majority of rs-fMRI literature has focused on group-level analyses, the challenge of rs-fMRI is to demonstrate utility at the subject level. Preliminary studies of motor mapping in presurgical mapping have yielded promising results, ${ }^{39}$ with motor-network extraction possible, even with the patient under anesthesia. ${ }^{40}$

Specific challenges are encountered when attempting to analyze rs-fMRI at the subject level. Mueller et al ${ }^{41}$ demonstrated that there is high intersubject variability in intrinsic brain networks generated from rs-fMRI, with differences in variability across cortical regions. For networks that are inherently variable across subjects, it will, therefore, be difficult to determine whether deviations from "known normal" connectivity of a particular network in an individual are related to that individual's specific connectivity profile or whether they are due to disease. In addition, functional connectivity may be altered depending on a subject's state, rather than trait. The optimum scanning length and parameters for reliable subject-level brain mapping using rs-fMRI are yet to be established.

These differences in acquisition parameters and processing/analysis pipelines may also yield vastly different results in rs-fMRI data. Head motion can introduce significant spurious functional correlations. ${ }^{42}$ Nuisance removal, which also is not standardized, may introduce errors. ${ }^{43}$ There is ongoing debate on the issue of instructing the subject to keep his or her eyes open or closed. Further work is necessary to determine a possible standardization, which would be important for using rs-fMRI as a clinically viable tool.

\section{CURRENT USE OF CVR IMAGING IN CLINICAL NEURORADIOLOGY \\ David Mikulis, MD; Department of Medical Imaging, University of Toronto, University Health Network, Toronto Western Hospital, Toronto, Ontario, Canada}

The high metabolic rate of the brain places significant demand on energy resources, even at resting levels of activity. ${ }^{44}$ The demand dramatically increases up to 3 -fold during functional activation. ${ }^{45}$ If the delivery of energy substrates, primarily oxygen and glucose, is not matched to metabolic demand, even for brief periods of time, functional impairment and even permanent injury can occur. A highly regulated mechanism has therefore evolved to control blood flow to match supply with demand. The flow-control mechanism achieves this by modulating the diameter of all arteries ranging from large arteries to arterioles. Most interesting, the capacity to dynamically modulate resistance increases with decreasing arterial size. ${ }^{46}$ Blood 

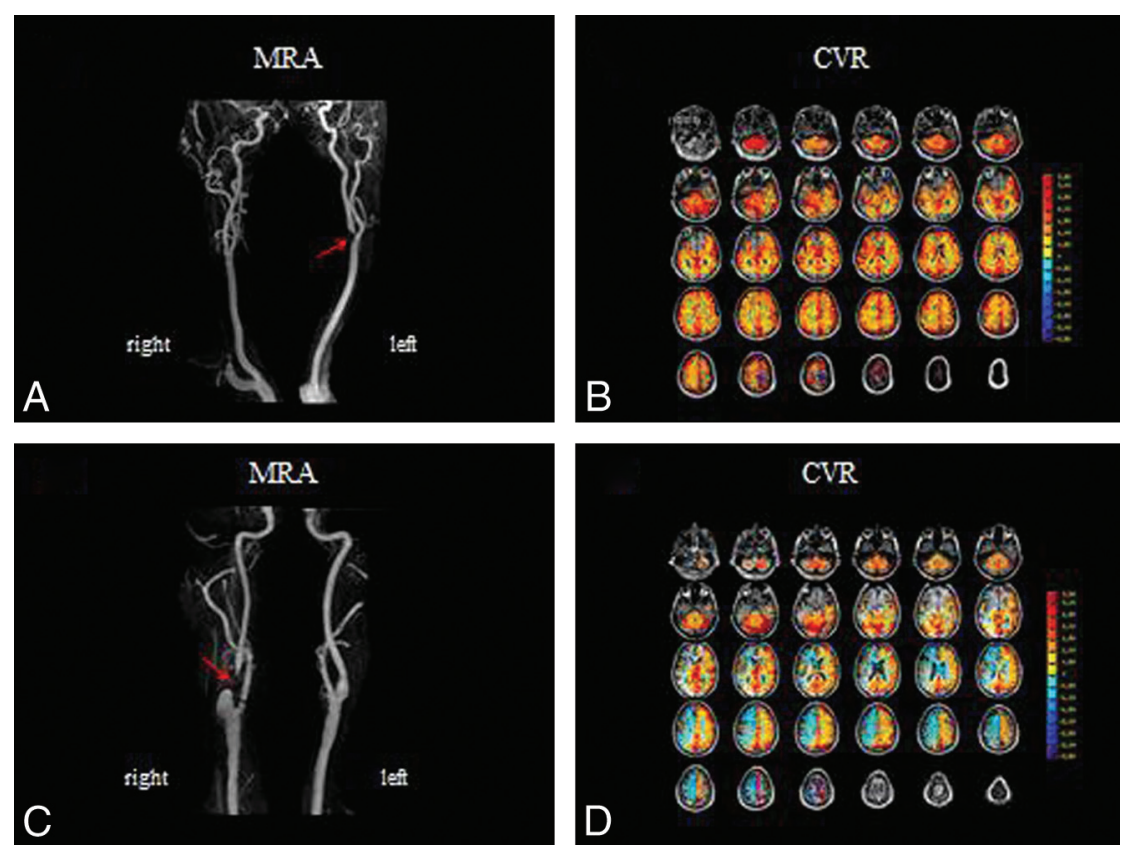

FIG 8. The results of CVR mapping by using a controlled elevation of arterial carbon dioxide during blood oxygen level-dependent MR imaging in 2 different patients presenting with transient ischemic attacks. Both patients ( $A$ and $C$ ) have $>90 \%$ carotid stenosis on MR angiography (red arrows). Corresponding CVR maps are shown with red/orange/yellow indicating increased oxyhemoglobin and therefore increased blood flow with $\mathrm{CO}_{2}$-induced vasodilation and blue indicating increased deoxyhemoglobin and therefore decreased blood flow with $\mathrm{CO}_{2}$-induced vasodilation. In the first patient, CVR is normal (B), indicating excellent collaterals, implying an embolic origin of symptoms. In the second patient $(D)$, the CVR map shows blue in the anterior circulation, indicating exhausted vascular reserve and steal physiology, implying that the symptoms are secondary to hemodynamic compromise. As opposed to the first patient, the second patient would not benefit from medical management alone requiring a flow-restoration procedure (endarterectomy or stent placement) to alleviate symptoms and stroke risk. Note that in both cases, findings of conventional perfusion imaging with MR imaging or CT by using dynamic bolus techniques would be abnormal, showing delays in transit time in the affected hemispheres.

flow to the microcirculation is, therefore, determined by 2 parameters: blood pressure and the overall resistance to flow in the upstream vasculature proximal to the capillary beds. The only caveats are the ability of capillary pericytes to contract ${ }^{47}$ and the resistance present in the venous vasculature.

Primary defects in the flow-control mechanism itself have not been clearly established, though dysregulation of flow has been implicated in diseases such as migraine, posterior reversible encephalopathy syndrome, reversible cerebral vasospasm syndrome, and pseudotumor cerebri, to name a few. The most common clinical condition in which the flow-control mechanism plays a key role is in cerebrovascular steno-occlusive disease most commonly caused by transient cerebral arteriopathy of childhood in the pediatric age group and by atherosclerosis in adults. The presence of a stenosis or occlusion leads to compensatory vasodilation in the arterial vasculature distal to the stenosis, preserving resting cerebral blood flow at normal levels. Because the ability to vasodilate is not infinite, the vasodilatory response to stenosis or occlusion may exhaust itself in the attempt to maintain normal resting $\mathrm{CBF}$. Under these circumstances, increased neuronal activity may not be met with increased blood flow. A mismatch between blood supply and demand ensues, which, by definition, represents ischemia unless it is matched by increased oxygen (and possibly glucose) extraction. Note that this does not represent failure of the flow-control mechanism per se. It simply indicates exhaustion of an appropriate response by a normal flow-control mechanism. Resting cerebral blood flow may be normal even when autoregulation is exhausted, indicating that detection of this condition may not be achievable by using resting blood flow measurement techniques such as arterial spin-labeling. However, flowmeasurement techniques that use bolus perfusion methods (perfusion CT and perfusion MRI) can yield, in addition to CBF, transit time and cerebral blood volume measures. Transit time and CBV will be abnormal when vascular reserve is exhausted, but they can also be abnormal with little or no exhaustion of vascular reserve if excellent collateral circulation has developed.

Exhaustion of vascular reserve can be detected by using any of several different modalities, including Doppler sonography, SPECT, xenon CT, perfusion CT, perfusion MRI, and blood oxygen leveldependent MR imaging. Each requires comparison of changes in blood flow before and after a vasodilatory stimulus. Is it important, however, to take this measurement if resting blood flow is normal? It is if one considers that ischemia in patients with exhausted reserve is a dynamic condition occurring at a time when the brain needs flow the most-during neuronal activation. This concept of dynamic ischemia under conditions with normal CBF is not usually a clinical consideration, though the potential consequences of this abnormal flow physiology were first described by Fisher. ${ }^{48} \mathrm{He}$ observed progressive dementia in patients with bilateral carotid occlusions in the absence of acute ischemic events. More recently, we observed the effects of this condition on cortical thickness. Thinning of the cerebral cortex was found in areas where the control mechanism was exhausted. ${ }^{49}$ Most surprisingly, rethickening was observed following revascularization. ${ }^{50}$ The implications are that the chronic ischemic events occurring during neuronal activation (ie, neurovascular uncoupling) lead to nonlethal injury to the neuropil (possibly decreased dendritic arborization and decreased synaptic density), which recovers following revascularization.

Although no pathologic studies have been performed to prove this hypothesis, the concept has been further supported by evidence of decreased $\mathrm{N}$-acetylaspartate acid levels (a marker of healthy neurons) in the cerebral cortex with exhausted vascular reserve (D.M., unpublished data). It is also supported by the Randomized Evaluation of Carotid Occlusion and Neurocognition (RECON) I study. ${ }^{51}$ In this study, patients with carotid occlusion, who were recruited for the Carotid Occlusion Surgery Study (COSS), ${ }^{52}$ were randomized into 2 groups: those with and those without an increased oxygen extraction fraction. A significant reduction in cognition was observed in the group with the increased 
oxygen extraction fraction. A follow-up study in these patients has not yet been reported, to our knowledge, but it is anticipated that cognitive improvement would be observed in those with an increased oxygen extraction fraction following extracranial-intracranial bypass compared with those treated medically. This finding could influence the direction of future research to more thoroughly examine the relationship between cognitive decline and cervicocerebral steno-occlusive disease. Measurement of vascular reserve would obviously play a key role in this regard.

Exhaustion of cerebrovascular reserve (CVR) can also be associated with steal phenomenon. It is now well-established that paradoxic reductions in blood flow can occur in tissues supplied by vessels with stenoses or occlusions during a vasodilatory stimulus. Studies have shown that steno-occlusive disease, when accompanied by steal physiology, carries up to 5 times the risk of ischemic injury. ${ }^{53}$ Despite this evidence, routine clinical application of CVR measurement is lacking. The reason for this is that current standards of practice have been based on trials (Asymptomatic Carotid Atherosclerosis Study and Asymptomatic Carotid Surgery Trial $)^{54,55}$ that advocate surgical management for asymptomatic carotid stenosis in excess of $70 \%$. Under these conditions, further testing is unnecessary because the added information would not alter management. Recent studies such as the Stenting and Aggressive Medical Management for Preventing Recurrent Stroke in Intracranial Stenosis (SAMMPRIS) trial, ${ }^{56}$ however, are beginning to show that aggressive or maximal medical management alone may match surgical outcomes. If under these circumstances, invasive treatment is only applied following failure in conservative management, then measurement of blood flow physiology remains moot.

So what is the clinical utility of CVR measurement? No prospective clinical trials have shown positive outcomes with measurement of vascular reserve embedded in management algorithms. Neither have there been positive clinical trials measuring resting blood flow parameters. However, in the future, 3 factors are likely to play an important role in proving the value of assessing flow physiology. The first is that proper selection of surgical candidates is critical for optimizing outcome. A criticism of the COSS study was that the oxygen extraction fraction thresholds used for selecting surgical candidates were too liberal so that the surgical group included a proportion of patients with less than severe flow deficits. ${ }^{57}$ Future clinical trials are needed to more fully elucidate this issue. The second factor is cost. Conservative management is 3 to 8 times more cost-effective than surgical management, ${ }^{58}$ again emphasizing the need for optimal selection strategies for costly invasive procedures. The final factor is the association of hemodynamic impairment with cognitive decline, especially if the decline can be shown to reverse after revascularization. The motivation to preserve or potentially improve cognition would clearly have profound effects on the standard of practice as it relates to caring for patients with steno-occlusive disease. Examples of the clinical use of CVR mapping are shown in Fig 8. These figures show the results of CVR by using a controlled elevation of arterial carbon dioxide during blood oxygen level-dependent MR imaging in 2 different patients presenting with transient ischemic attacks.

In summary, the importance of impaired blood flow control and blood flow hemodynamics has not yet entered mainstream management for patients with steno-occlusive diseases of the cervicocerebral vasculature. However, economics may eventually pressure the health care system into making more cost-effective choices, especially when the high annual costs of endarterectomies are considered. With 140,000 carotid endarterectomies performed in the United States in $2009^{59}$ and assuming $\$ 8000$ cost per operation, ${ }^{60}$ the cost to the health care system for endarterectomies alone is on the order of is $\$ 1.1$ billion annually. Considerable work toward finding optimal patient selection criteria is needed without compromising health care. Measurement of blood flow hemodynamics is a promising tool that could provide the important information for achieving this goal.

\section{REFERENCES}

1. Wang W, Qin W, Hao N, et al. Diffusion tensor imaging in spinal cord compression. Acta Radiol 2012;53:921-28

2. Sato T, Horikoshi T, Watanabe A, et al. Evaluation of cervical myelopathy using apparent diffusion coefficient measured by diffusion-weighted imaging. AJNR Am J Neuroradiol 2012;33:388-92

3. Mohamed F, Hunter L, Barakat N, et al. Diffusion tensor imaging of the pediatric spinal cord at $1.5 \mathrm{~T}$ : preliminary results. AJNR Am J Neuroradiol 2011;32:339-45

4. Barakat N, Mohamed F, Hunter L, et al. Diffusion tensor imaging of the normal pediatric spinal cord using an inner-field of view echoplanar imaging sequence. AJNR Am J Neuroradiol 2012;33:1127-33

5. Mulcahey MJ, Samdani A, Gaughan J, et al. Diffusion tensor imaging in pediatric spinal cord injury: preliminary examination of reliability and clinical correlation. Spine (Phila Pa 1976) 2012;37:E797-803

6. Jensen JH, Helpern JA, Ramani A, et al. Diffusional kurtosis imaging: the quantification of non-gaussian water diffusion by means of magnetic resonance imaging. Magn Reson Med 2005;53:1432-40

7. Lu H, Jensen JH, Ramani A, et al. Three-dimensional characterization of non-gaussian water diffusion in humans using diffusion kurtosis imaging. NMR Biomed 2006;19:236-47

8. Jensen JH, Helpern JA. Progress in diffusion-weighted imaging: concepts, techniques and applications to the central nervous system. NMR Biomed 2010;23:659-60

9. Raab P, Hattingen E, Franz K, et al. Cerebral gliomas: diffusional kurtosis imaging analysis of microstructural differences. Radiology 2010;254:876-81

10. Van Cauter S, Veraart J, Sijbers J, et al. Gliomas: diffusion kurtosis MR imaging in grading. Radiology 2012;263:492-501

11. Rosenkrantz AB, Sigmund EE, Johnson G, et al. Prostate cancer: feasibility and preliminary experience of a diffusional kurtosis model for detection and assessment of aggressiveness of peripheral zone cancer. Radiology 2012;264:126-35

12. Jensen JH, Falangola MF, Hu C, et al. Preliminary observations of increased diffusional kurtosis in human brain following recent cerebral infarction. NMR Biomed 2011;24:452-57

13. Hui ES, Fieremans E, Jensen JH, et al. Stroke assessment with diffusional kurtosis imaging. Stroke 2012;43:2968-73

14. Helpern JA, Adisetiyo V, Falangola MF, et al. Preliminary evidence of altered gray and white matter microstructural development in the frontal lobe of adolescents with attention-deficit hyperactivity disorder: a diffusional kurtosis imaging study. J Magn Reson Imaging 2011;33:17-23

15. Gao Y, Zhang Y, Wong CS, et al. Diffusion abnormalities in temporal lobes of children with temporal lobe epilepsy: a preliminary diffusional kurtosis imaging study and comparison with diffusion tensor imaging. NMR Biomed 2012;25:1369-77

16. Blockx I, Verhoye M, Van Audekerke J, et al. Identification and characterization of Huntington related pathology: an in vivo DKI imaging study. Neuroimage 2012;63:653-62

17. Grossman EJ, Ge Y, Jensen JH, et al. Thalamus and cognitive impairment in mild traumatic brain injury: a diffusional kurtosis imaging study. J Neurotrauma 2012;29:2318-27

18. Hori M, Fukunaga I, Masutani $Y$, et al. New diffusion metrics for 
spondylotic myelopathy at an early clinical stage. Eur Radiol 2012;22:1797-802

19. Prentice RI. Surrogate endpoints in clinical trials: definition and operational criteria. Stat Med 1989;8:431-40

20. Berger VW. Does the Prentice criterion validate surrogate endpoints? Stat Med 2004;23:1571-78

21. Ioannidis JP, Panagiotou OA. Comparison of effect sizes associated with biomarkers reported in highly cited individual articles and in subsequent meta-analyses. JAMA 2011;305:2200-10

22. Lu A, Atkinson IC, Claiborne T, et al. Quantitative sodium imaging with a flexible twisted projection pulse sequence. Magn Reson Med 2010;63:1583-93

23. Lu A, Atkinson IC, Thulborn KR. Sodium magnetic resonance imaging and its bioscale of tissue sodium concentration. In: Harris RK Wasylishen RE, eds. Encyclopedia of Magnetic Resonance. Hoboken, New Jersey: John Wiley; September 15, 2010.

24. Thulborn KR, Davis D, Snyder J, et al. Sodium MR imaging of acute and subacute stroke for assessment of tissue viability. Neuroimaging Clin N Am 2005 15:639-53

25. Thulborn KR, Lu A, Atkinson IC, et al. Quantitative sodium MR imaging and sodium bioscales for the management of brain tumors. Neuroimaging Clin N Am 2009;19:615-24

26. Thulborn KR. The challenges of integrating a 9.4T MR scanner for human brain imaging. In: Robitaille P-M, Berliner LJ, eds. Ultra High Field Magnetic Resonance Imaging. New York: Springer-Verlag Science and Business Media: 2006:105-26

27. Biswal B, Yetkin FZ, Haughton VM, et al. Functional connectivity in the motor cortex of resting human brain using echo-planar MRI. Magn Reson Med 1995;34:537-41

28. Beckmann CF, DeLuca M, Devlin JT, et al. Investigations into resting-state connectivity using independent component analysis. Philos Trans R Soc Lond B Biol Sci 2005;360:1001-13

29. van den Heuvel MP, Mandl RC, Kahn RS, et al. Functionally linked resting-state networks reflect the underlying structural connectivity architecture of the human brain. Hum Brain Mapp 2009;30:3127-41

30. Damoiseaux JS, Rombouts SA, Barkhof F, et al. Consistent restingstate networks across healthy subjects. Proc Natl Acad Sci U S A 2006; 103:13848-53

31. Van Dijk KR, Hedden T, Venkataraman A, et al. Intrinsic functional connectivity as a tool for human connectomics: theory, properties, and optimization. J Neurophysiol 2010;103:297-321

32. Fox MD, Snyder AZ, Zacks JM, et al. Coherent spontaneous activity accounts for trial-to-trial variability in human evoked brain responses. Nat Neuroc. 2006;9:23-5

33. Fox MD, Greicius M. Clinical applications of resting state functional connectivity. Front Syst Neurosci 2010;4:19

34. Spreng RN, Sepulcre J, Turner GR, et al. Intrinsic architecture underlying the relations among the default, dorsal attention, and fronto-parietal control networks of the human brain. J Cogn Neurosci 2013;25:74-86

35. Petrella JR, Sheldon FC, Prince SE, et al. Default mode network connectivity in stable vs progressive mild cognitive impairment. $\mathrm{Neu}$ rology 2011;76:511-17

36. Pievani M, de Haan $\mathrm{W}, \mathrm{Wu} \mathrm{T}$, et al. Functional network disruption in the degenerative dementias. Lancet Neurol 2011;10:829-43

37. Guo WB, Liu F, Chen JD, et al. Abnormal neural activity of brain regions in treatment-resistant and treatment-sensitive major depressive disorder: a resting-state fMRI study. J Psychiatr Res 2012;46:1366-73

38. Stufflebeam SM, Liu H, Sepulcre J, et al. Localization of focal epileptic discharges using functional connectivity magnetic resonance imaging. J Neurosurg 2011;114:1693-97

39. Zhang D, Johnston JM, Fox MD, et al. Preoperative sensorimotor mapping in brain tumor patients using spontaneous fluctuations in neuronal activity imaged with functional magnetic resonance imaging: initial experience. Neurosurgery 2009;65(6 suppl):226-36

40. Breshears JD, Gaona CM, Roland JL, et al. Mapping sensorimotor cortex with slow cortical potential resting-state networks while awake and under anestheia. Neurosurgery 2012;71:305-16

41. Mueller S, Wang D, Fox MD, et al. Individual variability in functional connectivity architecture of the human brain. Neuron 2013;77:586-95

42. Power JD, Barnes KA, Snyder AZ, et al. Spurious but systematic correlations in functional connectivity MRI networks arise from subject motion. Neuroimage 2012;59:2142-54

43. Hallquist MN, Hwang K, Luna B. The nuisance of nuisance regression: Spectral misspecification in a common approach to resting-state fMRI preprocessing reintroduces noise and obscures functional connectvity. Neuroimage 2013;82:208-25

44. Raichle ME, MacLeod AM, Snyder AZ, et al. A default mode of brain function. Proc Natl Acad Sci U S A 2001;98:676-82

45. Attwell D, Laughlin SB. An energy budget for signaling in the grey matter of the brain. Cereb Blood Flow Metab 2001;21:1133-45

46. Tuor UI, Farrar JK. Pial vessel caliber and cerebral blood flow during hemorrhage and hypercapnia in the rabbit. Am J Physiol 1984; 247(1 pt 2):H40-51

47. Hamilton NB, Attwell D, Hall CN. Pericyte-mediated regulation of capillary diameter: a component of neurovascular coupling in health and disease. Front Neuroenergetics 2010;2. pii

48. Fisher CM. Occlusion of the carotid arteries: further experiences. AMA Arch Neurol Psychiatry 1954;72:187-204

49. Fierstra J, Poublanc J, Han JS, et al. Steal physiology is spatially associated with cortical thinning. J Neurol Neurosurg Psychiatry 2010;81:290-93

50. Fierstra J, Maclean DB, Fisher JA, et al. Surgical revascularization reverses cerebral cortical thinning in patients with severe cerebrovascular steno-occlusive disease. Stroke 2011;42:1631-37

51. Marshall RS, Festa JR, Cheung YK, et al. Cerebral hemodynamics and cognitive impairment: baseline data from the RECON trial. Neurology 2012;78:250-55

52. Failure of extracranial-intracranial arterial bypass to reduce the risk of ischemic stroke: results of an international randomized trial-the EC/IC Bypass Study Group. N Engl J Med 1985;313:1191-200

53. Blaser T, Hofmann K, Buerger T, et al. Risk of stroke, transient ischemic attack, and vessel occlusion before endarterectomy in patients with symptomatic severe carotid stenosis. Stroke 2002;33:1057-62

54. Endarterectomy for asymptomatic carotid artery stenosis: Executive Committee for the Asymptomatic Carotid Atherosclerosis Study. J Am Med Assoc 1995;273: 1421-28

55. Halliday A, Mansfield A, Marro J, et al, for the MRC Asymptomatic Carotid Surgery Trial (ACST) Collaborative Group. Prevention of disabling and fatal strokes by successful carotid endarterectomy in patients without recent neurological symptoms: randomised controlled trial. Lancet 2004;363:1491-502. Erratum in Lancet 2004;364:416

56. Chimowitz MI, Lynn MJ, Derdeyn CP, et al, for the SAMMPRIS Trial Investigators. Stenting versus aggressive medical therapy for intracranial arterial stenosis. N Engl J Med 2011;365:993-1003

57. Yonas H, Carlson A, Nemoto EM. Carotid Occlusion Surgery Study (COSS): Failure of concept or methodology? In: Proceedings of the XXVth Symposium of International Society of Cerebral Blood Flow and Metabolism and the Xth International Conference on the Quantification of Brain Function with PET, Barcelona, Spain. May 25-28, 2011

58. Abbott AL. Medical (nonsurgical) intervention alone is now best for prevention of stroke associated with asymptomatic severe carotid stenosis: results of a systematic review and analysis. Stroke 2009;40:e573-83

59. National Hospital Discharge Survey 2009. Centers for Disease Control and Prevention. http://www.cdc.gov/nchs/nhds.htm. Accessed December 2010

60. Kilaru S, Korn P, Kasirajan K, et al. Is carotid angioplasty and stenting more cost effective than carotid endarterectomy? J Vasc Surg 2003;37:331-39 